\title{
Effects of 8-hour time restricted feeding on body weight and metabolic disease risk factors in obese adults: A pilot study
}

\author{
Kelsey Gabel ${ }^{\mathrm{a}}$, Kristin K. Hoddy ${ }^{\mathrm{a}}$, Nicole Haggerty ${ }^{\mathrm{a}}$, Jeehee Song ${ }^{\mathrm{a}}$, Cynthia M. Kroeger ${ }^{\mathrm{a}, \mathrm{b}}$, \\ John F. Trepanowski ${ }^{a}$, Satchidananda Panda ${ }^{\mathrm{c}}$ and Krista A. Varady ${ }^{\mathrm{a}, *}$ \\ ${ }^{a}$ Department of Kinesiology and Nutrition, University of Illinois at Chicago, Chicago, IL, USA \\ ${ }^{\mathrm{b}}$ School of Public Health, Indiana University, Bloomington, IN, USA \\ ${ }^{\mathrm{c}}$ Regulatory Biology Lab, Salk Institute for Biological Studies, La Jolla, CA, USA
}

\begin{abstract}
.
BACKGROUND: Time restricted feeding decreases energy intake without calorie counting and may be a viable option for weight loss. However, the effect of this diet on body weight in obese subjects has never been examined.

OBJECTIVE: This study investigated the effects of 8-h time restricted feeding on body weight and metabolic disease risk factors in obese adults.

DESIGN: Obese subjects $(n=23)$ participated in an 8-h time restricted feeding intervention (ad libitum feeding between 10:00 to 18:00 h, water fasting between 18:00 to 10:00 h) for 12 weeks. Weight loss and other outcomes were compared to a matched historical control group $(n=23)$.

RESULTS: Body weight and energy intake decreased in the time restricted group $(-2.6 \% \pm 0.5 ;-341 \pm 53 \mathrm{kcal} / \mathrm{d})$ relative to controls over 12 weeks $(P<0.05)$. Systolic blood pressure decreased in the time restricted feeding group $(-7 \pm 2 \mathrm{~mm} \mathrm{Hg})$ versus controls $(P<0.05)$. Fat mass, lean mass, visceral fat mass, diastolic blood pressure, LDL cholesterol, HDL cholesterol, triglycerides, fasting glucose, fasting insulin, HOMA-IR, and homocysteine were not significantly different from controls after 12 weeks (no group $\times$ time interaction).

CONCLUSIONS: These findings suggest that 8 -h time restricted feeding produces mild caloric restriction and weight loss, without calorie counting. It may also offer clinical benefits by reducing blood pressure.
\end{abstract}

Keywords: Time restricted feeding, intermittent fasting, body weight, metabolic disease risk factors, obese adults

\section{Introduction}

Intermittent fasting has gained considerable popularity over the past decade. There are two major subcategories of intermittent fasting: 1) fasting 1-4 d per week, i.e. alternate day fasting or the 5:2 diet [1]; or 2) fasting every day for a 14 to $20 \mathrm{~h}$ period, i.e. time restricted feeding $[2,3]$. Alternate day fasting and 5:2 are the most widely studied forms of inter-

\footnotetext{
*Corresponding author: Krista A. Varady, PhD, Associate Professor of Nutrition, Department of Kinesiology and Nutrition, University of Illinois at Chicago, 1919 West Taylor Street, Room 532, Chicago, 60612, IL, USA. Tel.: +1 312996 7897; E-mail: varady@uic.edu.
}

mittent fasting [4-10]. Human trials of alternate day fasting and 5:2 generally demonstrate reductions in body weight of 3 to $8 \%$ after 8 to 52 weeks of treatment, accompanied by decreases in blood pressure, LDL cholesterol, triglycerides, and insulin resistance [4-10]. The effects of time restricted feeding on the other hand, have only been tested in three human trials to date [11-13]. Gill and Panda [11] examined the effects of 10-h time restricted feeding in overweight healthy adults and showed a $4 \%$ weight loss that was sustained for one year. Findings from the other two studies $[12,13]$ reveal that $4-8 \mathrm{~h}$ time restricted feeding reduces caloric intake (without calorie counting) and significantly decreases fat mass without changing 
lean mass in young resistance trained men. While these preliminary studies offer promise for the use of time restricted feeding in reducing energy intake and fat mass, additional trials are necessary to confirm these findings.

Obesity greatly increases the risk of metabolic diseases, such a coronary heart disease and type 2 diabetes [14]. Accumulating evidence suggests that even small amounts of weight loss can lead to improvements in metabolic health [15]. Although alternate day fasting and 5:2 have been shown to be effective for weight loss [4-9], recent research suggests that obese individuals may have difficulties sticking to these diets long-term [10]. Time restricted feeding is an attractive alternative to alternate day fasting and 5:2, as this diet allows for ad libitum feeding within a large window of time each day, and does not require any calorie counting [2,3]. However, no trial to date has examined whether time restricted feeding is indeed an effective diet therapy for weight loss and metabolic disease risk reduction in obese subjects.

Accordingly, this study compared the effects of an 8-h time restricted feeding regimen versus a nointervention historical control group on body weight and metabolic disease risk factors in obese adults. We hypothesized that the time restricted feeding group would lose weight and improve blood pressure, plasma lipids, and glucoregulatory factors versus control subjects after 12 weeks of treatment.

\section{Methods}

\subsection{Time restricted feeding subject selection}

A 12-week trial was implemented to test the effects of time restricted feeding versus matched historical controls on body weight and metabolic disease risk factors. The experimental protocol was approved by the University of Illinois Chicago Office for the Protection of Research Subjects, and all research participants gave their written informed consent to participate in the trial. Time restricted feeding subjects were recruited from September 2016 to January 2017 from the Chicago area via advertisements placed around the University of Illinois Chicago campus. A total of 40 subjects were consented and assessed for eligibility to participate in the time restricted feeding intervention (Fig. 1). Of these 40 subjects, 11 subjects were excluded because they did not meet one or more inclusion criteria, and 6 subjects declined to participate after qualifying. Inclusion criteria was as follows: BMI between 30 and $45 \mathrm{~kg} / \mathrm{m}^{2}$; age between 25 and 65 years; pre-menopausal or post-menopausal (absence of menses for more than 2 years); sedentary to lightly active ( $<7500 \mathrm{steps} / \mathrm{d})$; weight stable for 3 months prior to the beginning of the study $(<4 \mathrm{~kg}$ weight loss or weight gain); non-diabetic; no history of cardiovascular disease (myocardial infarction or stroke); non-smoker; not a shift worker; and not taking weight loss, lipid- or glucose-lowering medications.

\subsection{Historical control subject selection}

Outcome measures were compared to a matched historical control group from a previous weight loss trial performed by our group from October 2011 to January 2015 [10] (Fig. 1). This previous trial implemented similar inclusion and exclusion criteria as the present trial, and all control subjects were recruited from the Chicago area via advertisements placed around the University of Illinois Chicago campus. There were 31 control subjects in the previous trial [10]. A stratified random sampling protocol based on age, BMI, and sex was used to match the historical control group $(n=23)$ to that of the time restricted feeding group $(n=23)$. Subjects were randomly selected from each consecutive strata, one-by one, until the sample size of 23 was reached.

\subsection{Baseline period}

Before commencing the study, both groups participated in a 2-week baseline weight stabilization period. All subjects were requested to remain weight stable by consuming their usual diet and not changing their physical activity habits. Body weight was measured weekly at the research center during this period.

\subsection{Time restricted feeding group protocol}

Time restricted feeding subjects were instructed to eat ad libitum from 10:00 to 18:00 h daily, and fast from 18:00 to 10:00 h daily. During the 8-h feeding window, there were no restrictions on types or quantities of foods consumed. Moreover, subjects were not required to monitor caloric intake during ad libitum feeding period. During the fasting period, subjects were encouraged to drink plenty of water and were permitted to consume energy-free beverages, such 

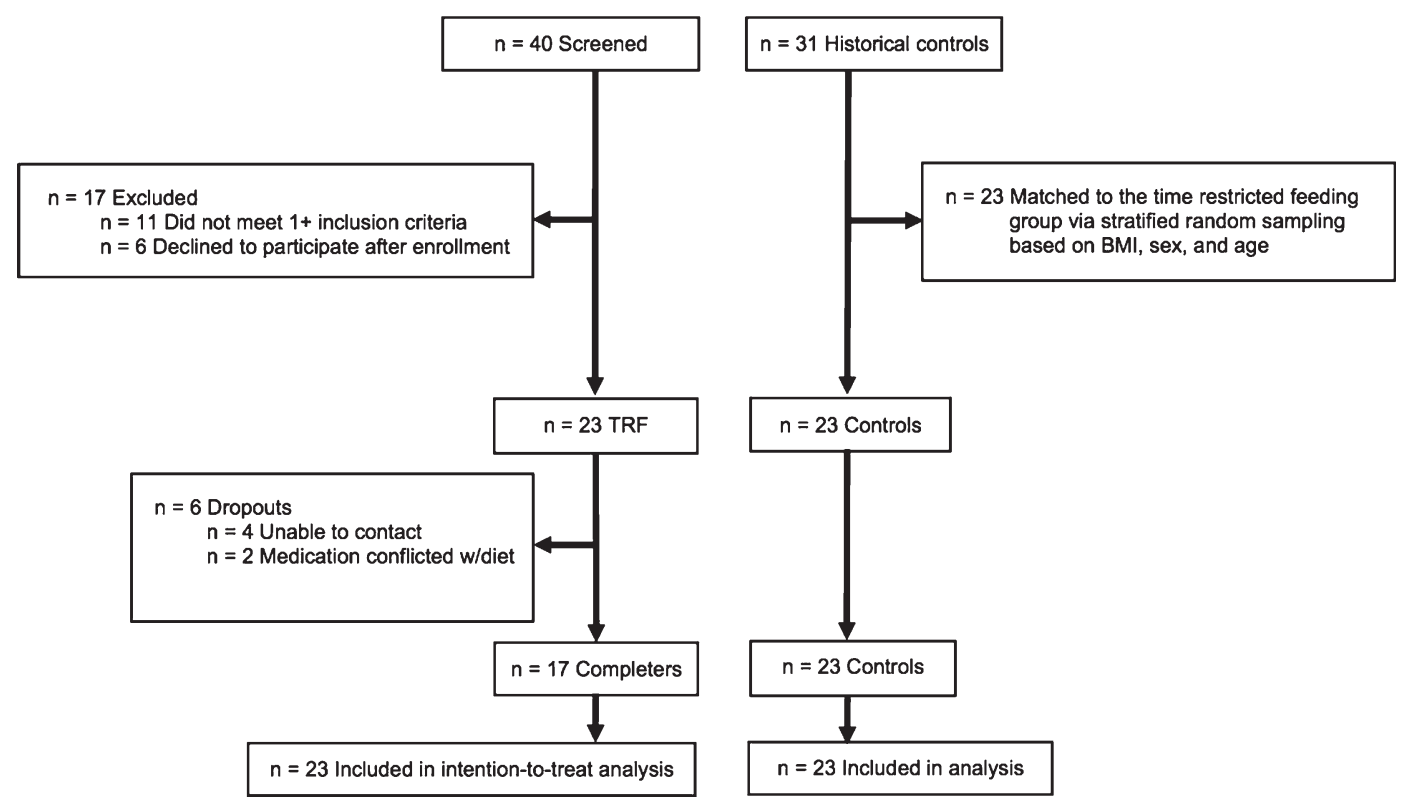

Fig. 1. Study flow chart.

black tea, coffee, and diet sodas. Adherence to the 8-h time restricted feeding window was measured using a daily adherence $\log$, which recorded the times each subject started and stopped eating each day. If the log indicated that the subject ate within the 8-h window (10:00 to 18:00 h), that day was labeled "adherent". If the log indicated that the subject consumed food outside of the 8-h feeding window, that day was labeled as "non-adherent". Adherence to the time restricted feeding diet was assessed as the number of adherent days per week.

\subsection{Historical control group protocol}

Historical controls were selected from a previous weight loss trial [10] performed by our group from 2011-2015. Controls were instructed to maintain their weight throughout the trial, and not to change their eating or physical activity habits. Controls visited the research center on a weekly basis for weigh-ins. Body composition and metabolic disease risk variables were assessed in control subjects every 12 weeks.

\subsection{Dietary intake and physical activity}

Time restricted feeding and control subjects completed a 7-d food record (on 7 consecutive days) during the baseline period and week 12. At base- line, a dietitian provided $15 \mathrm{~min}$ of instruction to each participant on how to complete the food records. These instructions included information and reference guides on how to estimate portion sizes and record food items in sufficient detail to obtain accurate estimates of dietary intake. Subjects were not required to weigh foods but were asked to measure the volume of foods consumed with household measures (i.e. measuring cups and measuring spoons). The timing of food intake was also recorded. Food records were collected at the weigh-in at baseline and week 12, and were reviewed by the dietitian for accuracy and completeness. The food analysis program, Nutritionist Pro (Axxya Systems, Stafford, TX) was used to calculate the total daily intake of energy, fat, protein, carbohydrate, cholesterol, and fiber. All subjects were asked to maintain their level of physical activity throughout the entire trial. We assessed activity level as steps/d. Step counts were measured over 7-d during the baseline period and at week 12 by a pedometer (Yamax Digi-walker SW-200, Yamax Inc., San Antonio, TX).

\subsection{Outcome measures}

\subsubsection{Body weight and body composition}

The primary outcome measure was body weight. Body weight was assessed to the nearest $0.25 \mathrm{~kg}$ every week without shoes and in light clothing using a balance beam scale (HealthOMeter, Boca Raton, FL). 
Height was assessed during the screening visit using a wall-mounted stadiometer (HealthOMeter, Boca Raton, FL) to the nearest $0.1 \mathrm{~cm}$. BMI was assessed as $\mathrm{kg} / \mathrm{m}^{2}$. Body composition (fat mass, lean mass, visceral fat mass) was measured using dual x-ray absorptiometry (DXA; iDXA, General Electric Inc).

\subsubsection{Metabolic disease risk factors}

Blood pressure and heart rate were measured at baseline and week 12 in triplicate using a digital automatic blood pressure/heart rate monitor (Omron HEM 705 LP, Kyoto, Japan) with the subject in a seated position after a 10 -min rest. Twelve-h fasting blood samples were collected between 5:00 and 9:00 $\mathrm{h}$ at baseline and week 12. The subjects were instructed to avoid exercise, alcohol, and coffee for $24 \mathrm{~h}$ before each visit. Blood was centrifuged for $10 \mathrm{~min}$ at $520 \mathrm{I} \mathrm{g}$ at $4^{\circ} \mathrm{C}$ to separate plasma from red blood cells and was stored at $-80^{\circ} \mathrm{C}$ until analyzed. Fasting plasma total cholesterol, direct LDL cholesterol, HDL-cholesterol, triglycerides concentrations were measured by a commercial lab (Alverno Laboratories, Hammond, IN). Fasting glucose concentrations were measured with a hexokinase reagent kit (Abbott, South Pasadena, CA). Fasting insulin was assessed as total immunoreactive insulin (Coat-ACount Insulin, Los Angeles, CA). Insulin resistance (IR) was calculated using the HOMA (Homeostasis Model Assessment) method, by applying the following formula: [HOMA-IR = Fasting insulin $(\mu \mathrm{lU} / \mathrm{ml}) \times$ Fasting glucose $(\mathrm{mg} / \mathrm{dL}) / 405]$. Plasma homocysteine was quantified using HPLC with fluorometric detection.

\subsection{Statistical analyses}

All data are presented as mean \pm standard error of the mean (SEM). Statistical analyses were performed using SPSS 24.0 for Windows (SPSS Inc.). A two-tailed $\mathrm{P}$ value of less than 0.05 was considered statistically significant. Tests for normality were included in the model, and all data were found to be normally distributed. For the sample size calculation, we estimated that time restricted feeding and control group would reduce body weight by $3 \%$ and $0 \%$, respectively, by week 12 . We calculated that $n=19$ participants per group would provide $80 \%$ power to detect a significant difference of $3 \%$ in body weight between the time restricted feeding and control group at week 12, using a two-tailed independent-samples $t$-test with $\alpha=0.05$. We anticipated a dropout rate of
$20 \%$. Thus, we aimed to recruit $n=23$ subjects in the time restricted feeding group, assuming that $n=19$ would complete the trial.

Differences between the time restricted feeding and control groups at baseline were analyzed by an independent samples $t$-test (continues variables) or the $\mathrm{McNemar}$ test (categorical variables). Data were included for 46 participants, and means were estimated using an intention-to-treat analysis using last observation carried forward. Repeated measures twofactor ANOVA with groups (time restricted feeding and control) as the between-subject factor and time (week 1 and 12) as the within-subject factor was used to compare changes in dependent variables between the groups over time. When there was a significant main effect but no interaction, post hoc comparisons were performed using Bonferroni's correction to determine differences between group means.

\section{Results}

\subsection{Baseline characteristics and dropouts}

As portrayed in Fig. 1, $n=23$ subjects began the time restricted feeding intervention and 6 dropped out. No subjects reported dropping out of the time restricted feeding group due to issues with the diet. At baseline, there were no statistically significant differences between the time restricted feeding group and the historical control group for age, sex, ethnicity, weight, height or BMI (Table 1).

Table 1

Baseline characteristics ${ }^{1}$

\begin{tabular}{lccc}
\hline & $\begin{array}{c}\text { Time restricted } \\
\text { feeding }\end{array}$ & Control & $P$ value $^{2}$ \\
\hline $\mathrm{N}$ & 23 & 23 & \\
Age (y) & $50 \pm 2$ & $48 \pm 2$ & 0.43 \\
Sex (F/M) & $20 / 3$ & $21 / 2$ & 0.82 \\
Ethnicity & 17 & & 0.69 \\
$\quad$ African American & 3 & 17 & \\
$\quad$ Caucasian & 3 & 5 & \\
$\quad$ Hispanic & $95 \pm 3$ & $92 \pm 3$ & 0.34 \\
Weight $(\mathrm{kg})$ & $1.66 \pm 0.02$ & $1.63 \pm 0.01$ & 0.29 \\
Height $(\mathrm{m})$ & $35 \pm 1$ & $34 \pm 1$ & 0.61 \\
BMI $\left(\mathrm{kg} / \mathrm{m}^{2}\right)$ & & & \\
\hline
\end{tabular}

${ }^{1}$ All values reported as mean $\pm \mathrm{SEM} .{ }^{2} P$ values for comparison of baseline variables between groups using independent samples $t$-test (continuous variables) and the McNemar test (categorical variables). 


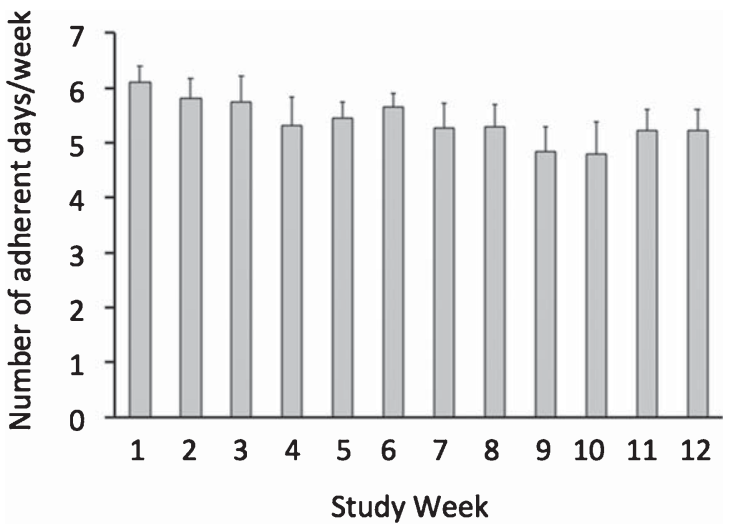

Fig. 2. Weekly adherence to the 8-hour feeding window by the time restricted feeding group. All values reported as mean \pm SEM. Each bar indicates the mean number of days per week that the time restricted feeding subjects were compliant with the 8-hour feeding window. On average, the time restricted feeding group was compliant with the prescribed eating window (10:00 to 18:00 h) on $5.6 \pm 0.3 \mathrm{~d} /$ week, and this level of adherence did not change over the course of the trial ( $P=0.86$, repeated measures ANOVA).

\subsection{Adherence to the time restricted feeding window}

Subjects in the time restricted feeding group were compliant with the prescribed eating window (10:00 to $18: 00 \mathrm{~h}$ ) on $5.6 \pm 0.3 \mathrm{~d}$ /week (Fig. 2), and this level of adherence did not change over the course of the trial $(P=0.86)$. At baseline, the time restricted feeding and control groups reported a similar mean daily eating duration of $11 \pm 1 \mathrm{~h}$ (Table 2). By week 12, mean daily eating duration in the time restricted feeding group was significantly shorter $(8 \pm 1 \mathrm{~h})$ than that of the control group $(11 \pm 1 \mathrm{~h}$ ) (group $\times$ time interaction, $P=0.01$ ). The start of the eating duration in the time restricted feeding group was later than that of the control group during the study (group $\times$ time interaction, $P=0.01$ ), and the end of the eating duration was similar between the groups during the study (no group $\times$ time interaction, $P=0.32$ ).

\subsection{Dietary intake and physical activity}

At baseline, energy intake was similar in the time restricted feeding and control groups (Table 2). During the trial, energy intake decreased in the time restricted feeding group by $341 \pm 53 \mathrm{kcal} / \mathrm{d}$ relative to controls (group $\times$ time interaction, $P=0.04$ ). Selfreported intake of macronutrients, dietary cholesterol and fiber did not differ between groups at baseline or post-treatment. Activity level, measured as steps/d, was similar at baseline in the time restricted feeding and control groups, and did not change over the course of the trial in either group.

\subsection{Body weight and body composition}

Body weight remained stable during the 2-week baseline period (Fig. 3). Body weight decreased in the time restricted group $(-2.6 \% \pm 0.5)$ relative to controls during the 12-week study (group $\times$ time interaction, $P<0.001)$. BMI decreased in the time restricted feeding group relative to the control group during the trial (group $\times$ time interaction, $P<0.001$ ) (Table 3 ). There were no statistically significant differences (i.e. no group $\times$ time interaction) between groups for fat mass, lean mass or visceral fat mass (Table 3).

\subsection{Metabolic disease risk factors}

At baseline, there were no differences between groups for any metabolic disease risk factor (Table 3). Systolic blood pressure significantly decreased in the time restricted feeding group $(-7 \pm 2 \mathrm{~mm} \mathrm{Hg})$ relative to controls during the study (group $\times$ time interaction, $P=0.02$ ). There were no statistically significant differences (i.e. no group $\times$ time interaction) between groups for diastolic blood pressure, heart rate, total cholesterol, LDL cholesterol, HDL cholesterol, triglycerides, glucose, insulin, HOMA-IR, or homocysteine.

\section{Discussion}

This study is the first trial to examine the impact of time restricted feeding on body weight and metabolic disease risk factors in an obese population. We show here that 12 weeks of 8 -h time restricted feeding (i.e. limiting food intake to 10:00 to $18: 00 \mathrm{~h}$ daily) decreases body weight by $\sim 3 \%$ relative to a no-intervention historical control group. We also demonstrate that this fasting regimen produces significant reductions in systolic blood pressure relative to controls.

Adherence to the time restricted feeding window was assessed daily via self-report. Our findings show that subjects were adherent to the prescribed eating window on $\sim 6$ days per week, and that this level of adherence remained constant throughout the 12week trial. On average, the time restricted feeding 
Table 2

Self-reported dietary intake, eating duration, and physical activity at baseline and week $12^{1}$

\begin{tabular}{|c|c|c|c|c|c|}
\hline & \multicolumn{2}{|c|}{ Time restricted feeding $(n=23)$} & \multicolumn{2}{|c|}{ Control $(n=23)$} & \multirow[t]{2}{*}{$P$-value Time $\times$ group $^{3}$} \\
\hline & Baseline $^{2}$ & Week 12 & Baseline $^{2}$ & Week 12 & \\
\hline Energy (kcal) & $1676 \pm 114$ & $1335 \pm 162$ & $1645 \pm 113$ & $1654 \pm 191$ & 0.04 \\
\hline Protein $(\%)$ & $16 \pm 1$ & $17 \pm 1$ & $17 \pm 1$ & $17 \pm 1$ & 0.40 \\
\hline Carbohydrates $(\%)$ & $47 \pm 2$ & $46 \pm 2$ & $46 \pm 2$ & $45 \pm 2$ & 0.61 \\
\hline Fat $(\%)$ & $37 \pm 1$ & $37 \pm 2$ & $37 \pm 1$ & $38 \pm 2$ & 0.74 \\
\hline Cholesterol (mg) & $279 \pm 24$ & $214 \pm 27$ & $275 \pm 27$ & $265 \pm 37$ & 0.32 \\
\hline Fiber $(\mathrm{g})$ & $16 \pm 2$ & $13 \pm 1$ & $14 \pm 1$ & $15 \pm 2$ & 0.17 \\
\hline Daily eating duration (h) & $11 \pm 1$ & $8 \pm 1$ & $11 \pm 1$ & $11 \pm 1$ & 0.01 \\
\hline Start of eating duration (local time, $\mathrm{h}$ ) & $8: 30 \pm 0: 30$ & $10: 00 \pm 0: 30$ & $9: 30 \pm 0: 30$ & $8: 30 \pm 0: 30$ & 0.01 \\
\hline End of eating duration (local time, $\mathrm{h}$ ) & $19: 30 \pm 0: 30$ & $18: 00 \pm 0: 30$ & $20: 30 \pm 0: 30$ & $19: 30 \pm 0: 30$ & 0.32 \\
\hline Steps/day & $6896 \pm 723$ & $7443 \pm 880$ & $6148 \pm 775$ & $6967 \pm 584$ & 0.84 \\
\hline
\end{tabular}

${ }^{1}$ All values reported as mean \pm SEM. Data for all variables were collected over a 7-d period at baseline (prior to the commencement of the study) and week 12 in the TRF and control groups. Data were included for 46 participants; means were estimated using an intention-to-treat analysis using last observation carried forward. ${ }^{2}$ Baseline variables: No difference between groups for any parameter (Independent samples $t$ test). ${ }^{3} P$ values reported for the time restricted feeding group relative to the control group (group $\times$ time interaction) using repeated-measures 2-factor ANOVA.

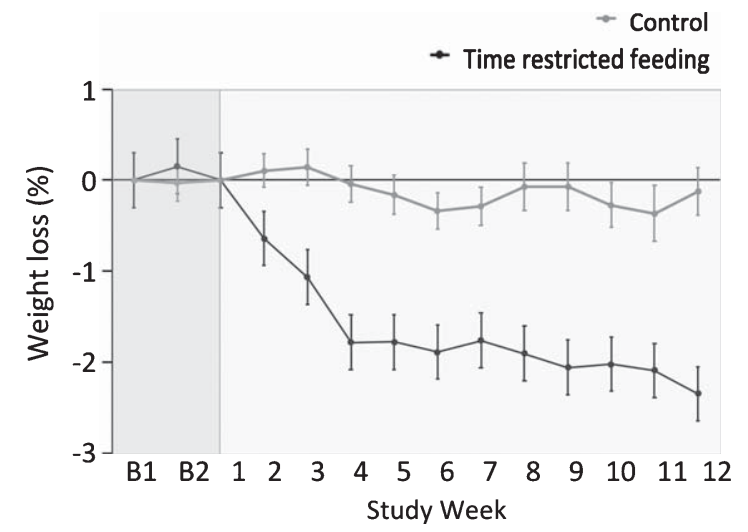

Fig. 3. Weight loss by the time restricted feeding group versus controls ${ }^{1} .{ }^{1}$ All values reported as mean \pm SEM. Data were included for 46 participants; means were estimated using an intention-totreat analysis using last observation carried forward. Body weight remained stable during the 2-week baseline period (week B1 and week B2). Body weight decreased in the time restricted feeding group relative to controls during the 12 -week intervention period $(P<0.001$ for time $\times$ group interaction $)$.

subjects reduced their daily eating duration by $3 \mathrm{~h} / \mathrm{d}$, i.e. from $11 \mathrm{~h} / \mathrm{d}$ to $8 \mathrm{~h} / \mathrm{d}$. The dropout rate in the time restricted feeding group (26\%) was high for a shortterm trial. However, no one in the time restricted feeding group reported dropping out due to issues with the diet. These preliminary findings suggest that time restricted feeding may be somewhat well tolerated over short periods in obese subjects.

Our findings also indicate that reducing the daily eating window to 8 -h/d decreases caloric intake by $\sim 300 \mathrm{kcal} / \mathrm{d}$, without intentional calorie counting.
As a result of this daily energy deficit, subjects in the time restricted feeding group lost $\sim 3 \%$ of body weight over 12 weeks, versus controls. The degree of weight loss demonstrated here is less than what was achieved in the 10-h time restricted feeding study (4\%) [11]. This may be due, in part, to the shorter trial duration (3 months) implemented here, when compared to the 10-h time restricted feeding study (4 months) [11]. Degree of energy restriction was also lower in the present study ( $\sim 300 \mathrm{kcal} / \mathrm{d}$ deficit), when compared to that of the 10-h time restricted feeding study ( $\sim 400 \mathrm{kcal} / \mathrm{d}$ deficit) [11]. Our results, however, are similar to the 4-8-h time restricted feeding trials which produced $\sim 1-3 \%$ body weight reductions $[12,13]$.

In comparison to other forms of intermittent fasting [4-10], time restricted feeding appears to produce less weight loss. For instance, after 12 weeks of alternate day fasting, body weight typically decreases by 4-6\% from baseline [4-10]. We speculate that this difference in weight loss is due to greater overall caloric restriction achieved with other forms of intermittent fasting, versus time restricted feeding. Accumulating evidence suggests that alternate day fasting produces an average caloric deficit of $25-35 \%$ daily $[10,16]$, whereas time restricted feeding may only produce a $20 \%$ caloric deficit daily [11]. The greater degree of energy restriction achieved with alternate day fasting is most likely the result of the vigilant calorie counting on fast days. Since time restricted feeding does not require subjects to monitor calorie intake at all, this may explain why the average caloric deficit achieved with time restricted feeding is lower. 
Table 3

Body composition and metabolic disease risk factors after 12 weeks ${ }^{1}$

\begin{tabular}{|c|c|c|c|c|c|}
\hline & \multicolumn{2}{|c|}{ Time restricted feeding $(n=23)$} & \multicolumn{2}{|c|}{ Control $(n=23)$} & \multirow[t]{2}{*}{$P$-value Time $\times$ group } \\
\hline & Baseline $^{2}$ & Week 12 & Baseline $^{2}$ & Week 12 & \\
\hline Body weight (kg) & $95 \pm 3$ & $92 \pm 3$ & $92 \pm 3$ & $92 \pm 3$ & $<0.001$ \\
\hline Fat mass $(\mathrm{kg})^{4}$ & $42 \pm 2$ & $40 \pm 2$ & $37 \pm 2$ & $37 \pm 2$ & 0.23 \\
\hline Lean mass $(\mathrm{kg})$ & $50 \pm 2$ & $50 \pm 2$ & $53 \pm 2$ & $53 \pm 2$ & 0.12 \\
\hline Visceral fat mass $(\mathrm{kg})$ & $1.2 \pm 0.1$ & $1.1 \pm 0.1$ & $1.2 \pm 0.2$ & $1.2 \pm 0.2$ & 0.19 \\
\hline $\operatorname{BMI}\left(\mathrm{kg} / \mathrm{m}^{2}\right)$ & $35 \pm 1$ & $34 \pm 1$ & $34 \pm 1$ & $34 \pm 1$ & $<0.001$ \\
\hline Systolic blood pressure (mm Hg) & $128 \pm 4$ & $121 \pm 3$ & $123 \pm 4$ & $124 \pm 3$ & 0.02 \\
\hline Diastolic blood pressure $(\mathrm{mm} \mathrm{Hg})$ & $83 \pm 2$ & $82 \pm 2$ & $81 \pm 2$ & $82 \pm 2$ & 0.41 \\
\hline Heart rate $(\mathrm{bpm})$ & $69 \pm 2$ & $71 \pm 2$ & $73 \pm 2$ & $73 \pm 3$ & 0.33 \\
\hline Total cholesterol (mg/dl) & $177 \pm 7$ & $178 \pm 9$ & $192 \pm 7$ & $185 \pm 7$ & 0.15 \\
\hline LDL cholesterol (mg/dl) & $108 \pm 5$ & $110 \pm 7$ & $114 \pm 7$ & $112 \pm 6$ & 0.54 \\
\hline HDL cholesterol (mg/dl) & $48 \pm 2$ & $49 \pm 2$ & $61 \pm 3$ & $55 \pm 2$ & 0.11 \\
\hline Triglycerides $(\mathrm{mg} / \mathrm{dl})^{4}$ & $105 \pm 11$ & $93 \pm 9$ & $89 \pm 7$ & $89 \pm 11$ & 0.43 \\
\hline Fasting glucose $(\mathrm{mg} / \mathrm{dl})$ & $79 \pm 4$ & $82 \pm 2$ & $87 \pm 2$ & $87 \pm 2$ & 0.77 \\
\hline Fasting insulin (uIU/ml) ${ }^{4}$ & $8.3 \pm 1.0$ & $5.7 \pm 0.7$ & $9.2 \pm 1.4$ & $10.3 \pm 1.9$ & 0.16 \\
\hline HOMA-IR & $1.6 \pm 0.2$ & $1.0 \pm 0.2$ & $2.0 \pm 0.3$ & $2.2 \pm 0.4$ & 0.21 \\
\hline Homocysteine $(\mu \mathrm{mol} / \mathrm{l})^{4}$ & $9.9 \pm 0.6$ & $9.0 \pm 0.5$ & $10.1 \pm 0.5$ & $9.4 \pm 0.5$ & 0.83 \\
\hline
\end{tabular}

${ }^{1}$ All values reported as mean \pm SEM. Data were included for 46 participants; means were estimated using an intention-to-treat analysis using last observation carried forward. HOMA-IR: Homeostatic model assessment Insulin resistance; RMR: Resting metabolic rate. ${ }^{2}$ Baseline variables: No difference between groups for any parameter (Independent samples $t$-test). ${ }^{3} P$ values reported for the time restricted feeding group relative to the control group (group $\times$ time interaction) using repeated-measures 2 -factor ANOVA. ${ }^{4}$ Significant main effect of time, $P<0.05$.

One potential confound in the current study is the lack of an objective measure to assess eating duration. As time restricted feeding is a recent concept, methods to objectively record eating time have yet to be optimized. Self-reporting of eating duration, as used in the current study, may not be optimal. An appbased recording of all eating events was used in the 10-h time restricted feeding study, and found a selfreporting error of $10 \%$ [11]. Objective methods of recording eating events show a mean eating duration that is different from what is widely believed [11]. A study assessing eating pattern among non-shift worker adults found the median daily eating duration can be 15 -h or longer, and less than $15 \%$ of adults eat for less than a 12-h duration [11]. In comparison, in the present study, the self-reported baseline eating duration was $11 \mathrm{~h}$, which is likely inaccurate. Our study also permitted the consumption of low energy drinks including coffee, tea, and diet soda. These drinks contain caffeine, which is known to perturb circadian rhythm [17]. Since time restricted feeding is based on the principle of circadian rhythm regulation of metabolism, low-energy caffeinated drinks may not count significantly towards energy consumption, but can have significant impact on circadian regulation.

Future trials in this area can be improved by using objective measures to better assess daily eating dura- tions. It will also be of interest to investigate whether shorter eating windows (4-6h) produce a degree of weight loss that is comparable to that of alternate day fasting and 5:2. Moreover, how the placement of the feeding window influences weight loss and adherence will also be important to examine. We chose to prescribe a feeding window of 10:00 to $18: 00 \mathrm{~h}$, so that the intervention would be standardized across all subjects. We assumed that this window would produce maximal adherence, as it would cause minimal disruption to the typical eating schedule (i.e. subjects could still have their breakfast in the morning, lunch in the early afternoon, and dinner in the early evening). However, recent trials $[18,19]$ have found that consuming larger meals earlier in the day produce better weight loss than similar sized meals consumed later in the evening. Whether weight loss and adherence can be improved by shifting the eating window earlier in the day warrants investigation.

Metabolic disease risk indicators remained relatively unaffected by the time restricted feeding regimen. Systolic blood pressure was the only parameter that improved over the course of the study, relative to controls. Reductions in insulin, insulin resistance, triglycerides, and homocysteine were also observed over time, but these effects were not statistically different from the control group. In the study by Moro et al. [12], plasma lipids 
and inflammatory factors also remained unchanged with 8-h time restricted feeding. It is likely that the degree of weight loss produced by 8 -h time restricted feeding was not large enough to improve these outcome measures. Accumulating evidence suggests that $>5 \%$ weight loss is required to improve plasma lipid concentrations and glucoregulatory factors [20]. It should also be noted that the obese subjects in the present study were metabolically healthy at baseline, i.e. their blood pressure, plasma lipid, glucose, and insulin levels were all within the normal range. Previous work indicates that intermittent fasting regimens $[4,8,10]$ and other lifestyle regimens $[21$, 22] have little effect on cardiometabolic disease risk factors in healthy obese subjects. It will be of interest to examine whether time restricted feeding can improve these risk factors in other groups of obese patients, such as those with compromised insulin sensitivity or dyslipidemia [23, 24].

This study has several limitations. First, the study was not a randomized controlled trial. We compared the effects of time restricted feeding to a matched historical control group from a previous weight loss trial conducted by our group. The trial [10] from which the controls were selected was conducted between 2011-2015. As such, the lapse of up to five years between trials could have influenced what the control subjects knew about weight control, and what foods were available in the marketplace due to seasonality. These issues should be considered when interpreting the present findings. In order to truly determine the effect of time restricted feeding on body weight and other metabolic disease variables, future trials should implement a randomized design where controls are enrolled concurrently. Second, the study was quite short (12 weeks). Longer-term trials will be needed to determine the degree of weight loss that can be achieved with time restricted feeding. Third, adherence and dietary intake were assessed by self-report, thus our estimates of eating duration and caloric deficit may be inaccurate [25, 26]. Implementing mobile apps to assess adherence in real-time [11] will help determine how well subjects can adhere to the prescribed eating window. Fourth, subjects completed a 7-d food record during the baseline period, which may have influenced their eating behaviors [27]. Recent findings suggest that subjects are more likely to report foods that are considered healthy and socially desirable, and omit foods that are considered unhealthy, when completing studyrelated food records [27]. Fifth, our study involved only metabolically healthy obese subjects, so our findings cannot be generalized to other population groups.

In summary, these findings suggest that 8-h time restricted feeding produces mild caloric restriction and weight loss in obese adults, without intentional calorie counting. This diet may also offer some clinical benefit by reducing systolic blood pressure. These preliminary data offer promise for the use of time restricted feeding as a weight loss technique in obese adults, but longer-term, larger-scale randomized controlled trials will be required before solid conclusions can be reached.

\section{Acknowledgments}

KG designed the research, conducted the clinical trial, analyzed the data, performed the statistical analysis, and wrote the manuscript; KKH, NH, JS, CMK, and JFT assisted with the conduction of the clinical trial; SP assisted with data interpretation and wrote the manuscript; KAV designed the research, analyzed the data, and wrote the manuscript.

\section{Funding}

University of Illinois Chicago Campus Research Board Pilot Grant; National Institutes of Health R01HL106228, F32DK107157, T32HL007909.

\section{Trial registration}

Clinicaltrials.gov NCT02948517.

\section{Disclosure}

Dr. Varady received author fees from Hachette Book Group for the book, "The Every Other Day Diet". Dr. Panda received author fees from Rodale Books for the upcoming book, "The Circadian Code". The other authors have no conflicts of interest to report.

\section{References}

[1] Longo VD, Mattson MP. Fasting: Molecular mechanisms and clinical applications. Cell Metab. 2014;19(2):181-92. 
[2] Longo VD, Panda S. Fasting, Circadian Rhythms, and Time-Restricted Feeding in Healthy Lifespan. Cell Metab. 2016;23(6):1048-59.

[3] Chaix A, et al. Time-restricted feeding is a preventative and therapeutic intervention against diverse nutritional challenges. Cell Metab. 2014;20(6):991-1005.

[4] Bhutani S, et al. Alternate day fasting and endurance exercise combine to reduce body weight and favorably alter plasma lipids in obese humans. Obesity (Silver Spring). 2013;21(7):1370-9.

[5] Catenacci VA, et al. A randomized pilot study comparing zero-calorie alternate-day fasting to daily caloric restriction in adults with obesity. Obesity (Silver Spring). 2016;24(9):1874-83.

[6] Harvie MN, et al. The effects of intermittent or continuous energy restriction on weight loss and metabolic disease risk markers: A randomized trial in young overweight women. Int J Obes (Lond). 2011;35(5):714-27.

[7] Heilbronn LK, et al. Alternate-day fasting in nonobese subjects: Effects on body weight, body composition, and energy metabolism. Am J Clin Nutr. 2005;81(1):69-73.

[8] Hoddy KK, et al. Meal timing during alternate day fasting: Impact on body weight and cardiovascular disease risk in obese adults. Obesity (Silver Spring). 2014;22(12): 2524-31.

[9] Klempel MC, Kroeger CM, Varady KA. Alternate day fasting (ADF) with a high-fat diet produces similar weight loss and cardio-protection as ADF with a low-fat diet. Metabolism. 2013;62(1):137-43.

[10] Trepanowski JF, et al. Effect of Alternate-Day Fasting on Weight Loss, Weight Maintenance, and Cardioprotection Among Metabolically Healthy Obese Adults: A Randomized Clinical Trial. JAMA Intern Med. 2017.

[11] Gill S, Panda S. A Smartphone App Reveals Erratic Diurnal Eating Patterns in Humans that Can Be Modulated for Health Benefits. Cell Metab. 2015;22(5):789-98.

[12] Moro T, et al. Effects of eight weeks of time-restricted feeding (16/8) on basal metabolism, maximal strength, body composition, inflammation, and cardiovascular risk factors in resistance-trained males. J Transl Med. 2016;14(1):290.

[13] Tinsley GM, et al. Time-restricted feeding in young men performing resistance training: A randomized controlled trial. Eur J Sport Sci. 2017;17(2):200-07.

[14] Jensen MD, et al. 2013 AHA/ACC/TOS guideline for the management of overweight and obesity in adults: A report of the American College of Cardiology/American Heart Association Task Force on Practice Guidelines and The Obesity Society. J Am Coll Cardiol. 2014;63(25 Pt B):2985-3023.

[15] Magkos F, et al. Effects of Moderate and Subsequent Progressive Weight Loss on Metabolic Function and Adipose Tissue Biology in Humans with Obesity. Cell Metab. 2016;23(4):591-601.

[16] Klempel MC, et al. Dietary and physical activity adaptations to alternate day modified fasting: Implications for optimal weight loss. Nutr J. 2010;9:35.

[17] Burke TM, et al. Effects of caffeine on the human circadian clock in vivo and in vitro. Sci Transl Med. 2015;7(305):305ra146.

[18] Garaulet M, et al. Timing of food intake predicts weight loss effectiveness. Int J Obes (Lond). 2013;37(4):604-11.

[19] Jakubowicz D, et al. High caloric intake at breakfast vs. dinner differentially influences weight loss of overweight and obese women. Obesity (Silver Spring). 2013;21(12): 2504-12.

[20] Ryan DH, Yockey SR. Weight Loss and Improvement in Comorbidity: Differences at 5\%,10\%, 15\%, and Over. Curr Obes Rep. 2017;6(2):187-94.

[21] Janiszewski PM, Ross R. Effects of weight loss among metabolically healthy obese men and women. Diabetes Care. 2010;33(9):1957-9.

[22] Kantartzis K, et al. Effects of a lifestyle intervention in metabolically benign and malign obesity. Diabetologia. 2011;54(4):864-8.

[23] Barnosky AR, et al. Intermittent fasting vs daily calorie restriction for type 2 diabetes prevention: A review of human findings. Transl Res. 2014;164(4):302-11.

[24] Harvie M, Howell A. Potential Benefits and Harms of Intermittent Energy Restriction and Intermittent Fasting Amongst Obese, Overweight and Normal Weight Subjects-A Narrative Review of Human and Animal Evidence. Behav Sci (Basel). 2017;7(1).

[25] Black AE, Cole TJ. Within- and between-subject variation in energy expenditure measured by the doubly-labelled water technique: Implications for validating reported dietary energy intake. Eur J Clin Nutr. 2000;54(5):386-94.

[26] Lichtman SW, et al. Discrepancy between self-reported and actual caloric intake and exercise in obese subjects. N Engl J Med. 1992;327(27):1893-8.

[27] Ortega RM, P.-R.C., Lopez-Sobaler AM. Dietary assessment methods: Dietary records. Nutr Hosp. 2015;31:38-45. 